\title{
Variables personales y sociales relacionadas con la dinámica bullying en escolares de Educación Primaria
}

\section{Consuelo Sánchez Lacasa y Fuensanta Cerezo Ramírez}

Departamento de Psicología Evolutiva y de la Educación, Universidad de Murcia

\section{España}

Correspondencia: Consuelo Sánchez Lacasa. Dpto. Psicología Evolutiva. Facultad de psicología. Campus de Espinardo. 30100. Murcia. España. E-mail: consuelosl@um.es

(C) Education \& Psychology I+D+i and Editorial EOS (Spain) 


\section{Resumen}

Introducción. Según el último informe del defensor del pueblo las conductas agresivas entre escolares ocurren frecuentemente en los centros educativos de nuestro país (España) y se dan en todas sus manifestaciones: insultos, amenazas, agresiones físicas, aislamiento social, etc., siendo en la actualidad cuando asistimos a un creciente interés por este fenómeno y los posibles factores de riesgo que inciden en el mismo. La edad y el sexo se recogen como los indicadores más estudiados, sin embargo ciertos autores indican la relación con otras variables personales tales como el presentar alguna discapacidad, la impulsividad o la nacionalidad de los escolares.

Método. El propósito de este estudio es describir la relación que existe entre los niveles de implicación en bullying en alumnos de Educación Primaria, ciertas variables personales y rechazo social. La muestra comprende un total de 426 alumnos escolarizados en los últimos cursos de Educación Primaria $\left(4^{\circ}, 5^{\circ}\right.$ y $\left.6^{\circ}\right)$ de tres centros públicos de la Región de Murcia (España). Se utilizó el cuestionario Test Bull-S para el análisis del nivel de implicación en bullying y el estudio de la dimensión sociométrica.

Resultados. Los resultados confirman que este fenómeno se da desde etapas más tempranas como es el caso de Educación Primaria. Asimismo, el estudio revela que existe relación entre ciertas variables personales y el fenómeno bullying, viéndose una implicación significativa de los chicos en todos los perfiles, de los sujetos con necesidades educativas específicas de apoyo educativo como víctimas, y de los sujetos repetidores e inmigrantes como agresores y víctimas-provocadoras.

Discusión y Conclusión. Ciertas variables personales (edad, sexo, necesidades educativas específicas de apoyo educativo, inmigración o repetición), en interacción con el rechazo social se perfilan como factores de riesgo para la implicación en bullying. Desde esta perspectiva del fenómeno vemos necesaria una amplia evaluación que describa los sujetos implicados directamente y perfile los aspectos personales y contextuales que pueden estar incidiendo en su aparición y mantenimiento desde las primeras edades.

Palabras Clave: agresor, víctima, víctima-provocadora, necesidades específicas de apoyo educativo, inmigración, repetición, rechazo social, preadolescentes.

Recibido: 31/05/10 Aceptación Inicial: 07/06/10 Aceptación Definitiva: 08/07/10 


\title{
Personal and social characteristics of schoolchildren in- volved in bullying in primary education
}

\begin{abstract}
Introduction. According to the latest report of the ombudsman the aggressive behaviours happen frequently in the schools in our country (Spain) and it appears in all its manifestations: insults, threats, physical aggression, social isolation, etc., but it is now when we are witnessing a growing interest in this phenomenon and the possible risk factors to help understand and prevent these behaviors from early childhood. Individual risk factors such as age and sex have been well documentated however other studies indicate other personal variables as disabilities, impulsivity or nationality.
\end{abstract}

Method. The purpose of this study is to describe the relationship between levels of involvement in bullying in primary school children, some personal variables and social rejection. The study includes a total of 426 students enrolled in the last years of primary school (4th, 5th and 6th) from three public schools in the Region of Murcia (Spain). Bull-S Test questionnaire was used for the analysis of the level of involvement in bullying and the study of sociometric dimension.

Results. The results confirm that this phenomenon is happening at younger ages, particulary at Primary School. The study also reveals that there is a relationship between certain personal variables (age, gender, specific needs of educational support, immigration and repetition) and the bullying, finding a significant involvement of children in all profiles, of children with specific needs of educational support as victims and repeaters and immigrant subjects as bullies and bully-victims.

Discussion and Conclusion. Certain personal variables (age, gender, specific needs of educational support, immigration and repetition) in interaction with social rejection, emerge as risk factors for involvement in bullying. From this perspective, the phenomenon require an evaluation describing the subjects directly involved in bullying behaviors and outlines the contextual aspects that may be affecting and supporting it.

Keywords: bully, victim, bully-victim, specific needs of educational support, inmigration, repetition, social rejection, preadolescence.

Received: 05/31/10 Initial Acceptance: 06/07/10 Definitive Acceptance: 07/08/10 


\section{Introducción}

En nuestro país, son varios los estudios, que nos aportan información sobre la incidencia del fenómeno bullying en la etapa de Educación Secundaria, poniendo de manifiesto todos ellos que este fenómeno está presente en esta etapa educativa y con porcentajes considerables (Avilés y Monjas, 2005; Defensor del Pueblo, 2007; Serrano e Iborra, 2005). El interés está puesto en detectar estas situaciones en edades más tempranas encontrando diversos estudios que confirman que se da una "ampliación hacia los extremos" que hace pensar que el fenómeno bullying se va generalizando a todas las edades escolares (Cerezo, 2009; Cerezo, Calvo y Sánchez, 2004; Craig y Pepler, 2007; Wolke, Woods, Stanford y Schulz, 2001).

Entre los factores de riesgo individuales, algunos estudios de Inglaterra apuntan hacia la edad y el sexo como primeros indicadores relacionados con la incidencia del fenómeno, aunque cada vez más dirigen la atención hacia otras características personales como el nivel de inteligencia, presentar alguna discapacidad, la impulsividad o la nacionalidad (Farrington, 2005; Smith, 2004; Woods, Hall, Dautenhadn y Wolke, 2007).

En relación al sexo, los estudios indican que son los chicos los que se ven implicados con mayor frecuencia en el fenómeno bullying, observándose tasas más altas como agresores y como víctimas (Cerezo, 2010; Postigo, González, Mateu, Ferrero y Martorell, 2009; Solberg y Olweus, 2003; Tapper y Boulton, 2005). Sin embargo, otros estudios señalan que las diferencias por sexo en la dinámcia bullying están marcadas especialmente en las agresiones directas como la violencia física o las amenazas, mientras que están menos marcadas en las agresiones indirectas como los rumores, el aislamiento o el rechazo (González, Postigo, Montoya y Martorel, 2007; Toldos, 2005; Veensta, Lindenberg, De Winter, Verhulst y Ormel, 2005).

Con respecto a la edad se apunta la necesidad de continuar investigando la relación con la implicación en bullying (Benítez y Justicia, 2006), encontrando pocos estudios de tipo longitudinal. Un estudio retrospectivo dirigido por Eslea y Rees (2001), afirmaba que la edad en la que se concentra un mayor número de víctimas es la que va desde los 11 años a los 13 años. Otros autores, sin embargo, recogen que el máximo se da entre los 9 y los 15 e indican que los agresores son mayores que sus víctimas (Carney y Merrell, 2001; Seals y Young, 2003). Además, los estudios realizados en nuestra región (Murcia), ponen de manifiesto que 
actúan como agresores los sujetos que están escolarizados en grupos de edad inferiores a la suya, apareciendo el efecto de la repetición como elemento decisivo en estas situaciones (Cerezo, 2006).

En relación a los alumnos que presentan necesidades específicas de apoyo educativo, cabe señalar que los sujetos con deficiencia mental pueden verse más implicados como víctimas, ya que suelen estar menos integrados socialmente, no tienen protección de sus iguales y presentan una actitud pasiva ante las actuaciones de los agresores (Card, 2003). Por su parte, Farrington (2005) señala que los alumnos con un cuadro de "hiperactividad-impulsividaddéficit de atención" son los que presentan los factores de personalidad más importantes para predecir un comportamiento antisocial y por lo tanto verse implicados en situaciones de agresividad. Otros estudios señalan que los agresores tienen mayor tendencia a presentar déficit en las funciones ejecutivas y establecen relación entre el déficit de atención e hiperactividad y bullying (Mestre, Samper y Frías, 2002; Unnever y Cornell, 2003). Estos alumnos podrían verse involucarados como víctimas-provocadoras por tener pocos amigos y bajo estatus social, respondiendo de forma impulsiva a la hora de defenderse.

Otro de los factores individuales relacionado con la dinámica bullying es la nacionalidad. Al parecer, el pertenecer a minorías étnicas o ser inmigrante es un factor de riesgo para verse involucrado en estas situaciones, aunque lo que no está claro es el perfil de éstos. A este respecto, los estudios de Unnever y Cornell (2003) apuntan que los sujetos inmigrantes se encuentran involucrados con mayor frecuencia como agresores mientras que Wolke, Woods, Stanford y Schulz (2001) señalan que los sujetos de otras nacionalidades se ven implicados como víctimas, siendo el insulto racista el tipo de agresión más padecida. En nuestro país (España), el último Informe del Defensor del Pueblo (2007), también pone de manifiesto la incidencia de los sujetos inmigrantes en el fenómeno bullying, indicando que éstos aparecen involucrados como víctimas o como testigos, mientras que Cerezo, Calvo y Sánchez (2004) recogen una implicación como víctimas y víctimas-provocadoras (v-p).

Si todos estos aspectos individuales han sido relacionados con la implicación en bullying, también es cierto que existe cierta relación entre ellos, la competencia social y los aspectos relacionales del aula. Es así, que los profesores evalúan a los niños menos habilidosos socialmente que a las niñas (Díaz-Aguado, 2005) y relacionan a los chicos con deficiencia mental con más conductas de aislamiento e impopularidad (Trianes, Blanca, García y Sánchez, 
2003). Del mismo modo, algunos estudios señalan el rechazo social como factor de riesgo para verse implicado en las conductas bullying e indican que es un problema de relación que tiene lugar dentro del entramado social del aula (Craig y Pepler, 2007; Gifford-Smith y Brownell, 2003).

\section{Objetivos e hipótesis}

El estudio recoge un doble objetivo: a) describir la incidencia del fenómeno bullying en sujetos de 9-12 años y b) analizar la relación entre ciertas variables individuales y la incidencia del fenómeno bullying tales como la edad, el sexo, ser un alumno con necesidades específicas de apoyo educativo (n.e.a.e), ser un sujeto inmigrante y ser un sujeto repetidor, así como ver la relación entre éstas y el rechazo social. Estos objetivos se concretan en las siguientes hipótesis:

1. El fenómeno bullying está presente en todos los niveles y edades del estudio.

2. Ciertas variables personales y sociodemográficas, relacionadas con baja integración social, favorecen la implicación en bullying:

- 1.1. Los chicos se ven más implicados que las chicas en todos los perfiles.

- 1.2. Los sujetos inmigrantes y con n.e.a.e se ven más implicados como víctimas y víctimas-provocadoras.

- 1.3. Los sujetos repetidores se ven más implicados como agresores.

\section{Método}

\section{Participantes}

Se seleccionaron tres centros públicos de la Región de Murcia (España), ubicados dos de ellos en localidades rurales y uno en el centro de la ciudad. La muestra está formada por 426 sujetos de edades comprendidas entre 9 y 12 años que se encontraban matriculados en los últimos cursos de Educación Primaria $\left(4^{\circ}, 5^{\circ}\right.$ y $\left.6^{\circ}\right)$ en el momento de la recogida de información. 
El estudio comprende un total de 18 aulas (un $20 \%$ de los sujetos cursan el nivel de $4^{\circ}$, un $38 \% 5^{\circ}$ y un $42,3 \% 6^{\circ}$ de Educación Primaria). La distribución por edad es de $10 \%$ de sujetos de 9 años, un 35,7\% de 10 años, un $41 \%$ de 11 años y un $13 \%$ de 12 años. En cuanto al sexo nos encontramos que una vez seleccionada la muestra, un $48 \%$ de la muestra son chicos y un 51,4\% chicas, por lo que ambos sexos se encuentran representados con porcentajes similares. Los tres centros son de grandes dimensiones contando con 2 y 3 aulas por nivel, por lo que nos encontramos con la designación de grupos A, B y C. En la Tabla 1 se resumen las características de la muestra, configurando cada una de ellas las varibles independientes del estudio.

Tabla 1. Características de la muestra

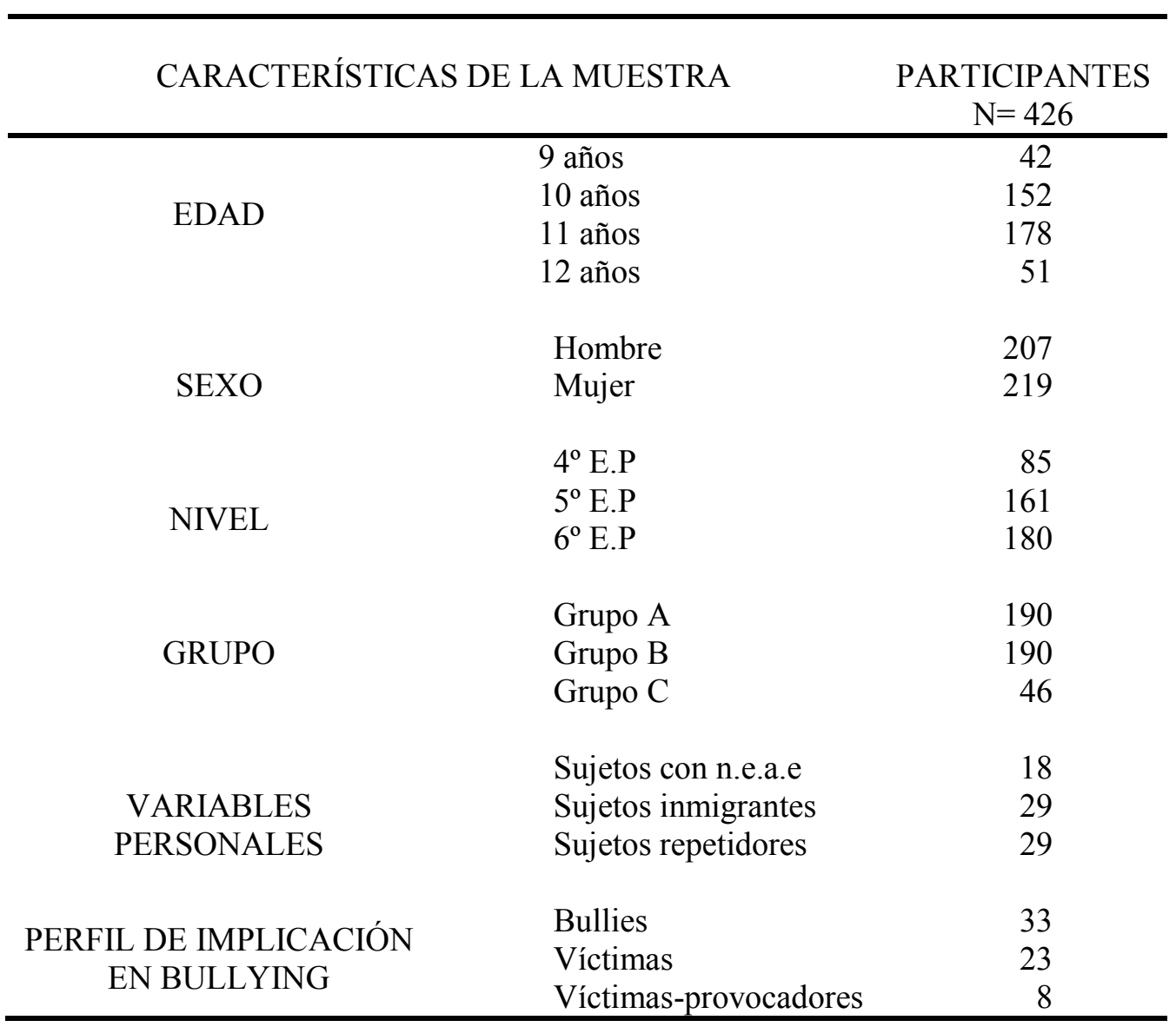

\section{Instrumentos}

Para el estudio se ha utilizado el cuestionario Test Bull-S (Cerezo, 2002) cuyo análisis interpretativo nos ofrece información sobre la estructura del grupo y la relación agresiónvictimización a través de dos dimensiones que engloba las variables dependientes del estudio. 
1. La dimensión sociométrica está formada por variables que definen la posición social de cada miembro y la estructura socio-afectiva del grupo en su conjunto a través de los ítems a los que el sujeto responde con el nombre de tres compañeros: 1. Elegido, 2. rechazado, 3. Expectativa de ser elegido y 4. Expectativa de ser rechazado.

2. La dimensión de la Dinámica bullying está definida por las variables sobre la relación Agresión-Victimización, distinguiendo entre las específicas para el agresor y la víctima (ítem 5 a 10): 5. Fuerte, 6. Cobarde, 7. Agresivo, 8. Victima, 9. Provoca, 10. Manía. Los ítems correspondientes a estas dos dimensiones se presentan en forma de nominación o elección entre los sujetos del grupo y a través de estas variables se comprueban las características asociadas al perfil del agresor (fuerte, agresivo y provocador) y las características asociadas al perfil de la víctima (cobarde, víctima y manía). Esta dimensión nos permite establecer el nivel de implicación en bullying a través de cuatro valores:

- $0=$ no implicados en bullying (otros)

- 1 = bullies (nominados por al menos el 25\% como agresor)

- 2 = víctimas (nominados por al menos el 25\% como víctima)

- 3 = víctima-provocadora (nominado por al menos el $25 \%$ como agresor y como víctima).

Los niveles de fiabilidad se obtuvieron a través del cálculo del Coeficiente Alfa entre los ítems del cuestionario, donde los resultados mostraron niveles de fiabilidad satisfactorios que permitieron concluir que el cuestionario Bull-S es un instrumento fiable y válido para medir la agresividad entre escolares (Alfa de Cronbach para $\mathrm{N}=322$, fue de .73).

\section{Procedimiento}

El proceso de recogida de datos se inició con la selección de los centros de Educación Infantil y Primaria de la Región de Murcia. En un primer momento, se contactó con los equipos directivos con el objeto de explicarles los objetivos y el alcance de la investigación, así como proponerles su participación voluntaria. Una vez, que la dirección de los centros manifestó su consentimiento de colaboración, se realizó una reunión informativa con los tutores de los cursos implicados en la muestra, con el fin de explicar el objetivo de nuestro estudio y recoger información relevante de los respectivos cursos. Paralelamente se remitió a los padres una carta explicativa de la investigación para obtener su consentimiento acerca de la participación de su hijo/a en el estudio. 
Tras acordar la participación de profesores y alumnos/as, se establecieron los días para la realización de las diferentes pruebas, de manera que los sujetos de cada aula cumplimentaron los cuestionarios en sus respectivas aulas en dos sesiones diferentes. Para coordinar y revisar la aplicación de los instrumentos en las diferentes aulas se contó con la colaboración de varios investigadores previamente formados.

Los alumnos fueron informados en la primera sesión de la importancia del objeto del estudio y de las implicaciones educativas, obteniendo una buena participación. Se garantizó el anónimato de los datos y una vez cumplimentadas las diferentes pruebas, éstas se introdujeron en un sobre que se cerró delante de ellos, anotando el nombre del colegio, el nivel y el grupo para su identificación.

\section{Diseño y análisis estadístico}

Dentro de un diseño de investigación empírico cuantitativo, se ha realizado por un lado un estudio descriptivo de la incidencia del fenómeno bullying, pero también se define como un estudio "ex post facto" retrospectivo de un grupo con múltiples medidas, que nos ha permitido estudiar la relación entre las variables independientes y la variable dependiente, utilizando los cuestionarios para la obtención de evidencia empírica (Montero y León, 2008). Para el análisis de la incidencia de la dinámica bullying se ha realizado un estudio de frecuencias en el conjunto de la muestra y para establecer si existen diferencias significativas en función de las variables independientes se ha realizado la prueba chi-cuadrado $\left(\chi^{2}\right)$. También se ha comprobado el tamaño del efecto a través de los índices de asociación "coeficiente Phi” y “coeficiente V de Cramer", recogiéndose en las variables cuya magnitud alcanza un valor mínimo de 0.10. Para ver la relación entre las variables personales y el rechazo social se realizó un análisis T-Test Bull-S (Prueba T para muestras independientes) que permitió establecer comparaciones dos a dos de los subgrupos entre las variables individuales y la dimensión sociométrica. 


\section{Resultados}

\section{Incidencia del fenómeno bullying}

En el análisis por nivel, los datos recogidos en la tabla 2 muestran que el fenómeno bullying está presente en todos los niveles del estudio y además no se observan diferencias significativas entre ellos. Si bien, cabe destacar que el porcentaje de implicación en $5^{\circ}$ y $6^{\circ}(17 \%$ y $15 \%)$ es algo mayor que en $4^{\circ}(12 \%)$.

Por grupos, se observa que el fenómeno bullying está presente en todos y no resultan significativas las diferencias entre ellos, si bien se aprecia en la tabla 2 un porcentaje más elevado en el grupo C (24\%), donde se observa una implicación del 11\% como agresores y del 6,5\% como víctimas-provocadoras.

Tabla 2. Frecuencias por nivel, grupo y edad. Porcentajes y valores absolutos

\begin{tabular}{|c|c|c|c|c|c|c|c|c|c|c|}
\hline & \multicolumn{3}{|c|}{ NIVEL } & \multicolumn{3}{|c|}{ GRUPO } & \multicolumn{4}{|c|}{ EDAD } \\
\hline & $4^{\circ}$ & $5^{\circ}$ & $6^{\circ}$ & $\mathbf{A}$ & B & C & 9 & 10 & 11 & 12 \\
\hline OTROS & $\begin{array}{l}88,2 \\
(75)\end{array}$ & $\begin{array}{c}83,2 \\
(134)\end{array}$ & $\begin{array}{c}85,2 \\
(153)\end{array}$ & $\begin{array}{c}85,8 \\
(163)\end{array}$ & $\begin{array}{c}81,3 \\
(164)\end{array}$ & $\begin{array}{l}76,1 \\
(35)\end{array}$ & $\begin{array}{l}90,5 \\
(38)\end{array}$ & $\begin{array}{c}84,9 \\
(129)\end{array}$ & $\begin{array}{l}84,3 \\
(150)\end{array}$ & $\begin{array}{l}83,3 \\
(45)\end{array}$ \\
\hline BULLY & $\begin{array}{l}5,9 \\
(5)\end{array}$ & $\begin{array}{l}8,7 \\
(14)\end{array}$ & $\begin{array}{c}7,8 \\
(14)\end{array}$ & $\begin{array}{l}7,9 \\
(15)\end{array}$ & $\begin{array}{c}6,8 \\
(13)\end{array}$ & $\begin{array}{c}10,9 \\
(5)\end{array}$ & $\begin{array}{l}4,8 \\
(2)\end{array}$ & $\begin{array}{l}7,2 \\
(11)\end{array}$ & $\begin{array}{c}7,9 \\
(14)\end{array}$ & $\begin{array}{l}11 \\
(6)\end{array}$ \\
\hline VÍCTIMA & $\begin{array}{l}3,5 \\
(3)\end{array}$ & $\begin{array}{l}5,6 \\
(9)\end{array}$ & $\begin{array}{c}6,1 \\
(11)\end{array}$ & $\begin{array}{l}4,2 \\
(8)\end{array}$ & $\begin{array}{c}6,3 \\
(12)\end{array}$ & $\begin{array}{l}6,5 \\
(3)\end{array}$ & $\begin{array}{l}4,8 \\
(2)\end{array}$ & $\begin{array}{l}5,3 \\
(8)\end{array}$ & $\begin{array}{c}6,2 \\
(11)\end{array}$ & $\begin{array}{l}3,7 \\
(2)\end{array}$ \\
\hline V-P & $\begin{array}{l}2,4 \\
(2)\end{array}$ & $\begin{array}{l}2,5 \\
(4)\end{array}$ & $\begin{array}{l}1,1 \\
(2)\end{array}$ & $\begin{array}{c}2 \\
(4)\end{array}$ & $\begin{array}{l}0,5 \\
(1)\end{array}$ & $\begin{array}{l}6,5 \\
(3)\end{array}$ & - & $\begin{array}{l}2,6 \\
(4)\end{array}$ & $\begin{array}{l}1,7 \\
(3)\end{array}$ & $\begin{array}{l}1,9 \\
(1)\end{array}$ \\
\hline TOTAL BULLYING & $\begin{array}{l}11,8 \\
(10)\end{array}$ & $\begin{array}{l}16,8 \\
(23)\end{array}$ & $\begin{array}{c}15 \\
(27)\end{array}$ & $\begin{array}{l}14,2 \\
(27)\end{array}$ & $\begin{array}{l}13,6 \\
(26)\end{array}$ & $\begin{array}{l}23,9 \\
(11)\end{array}$ & $\begin{array}{c}9 \\
(4)\end{array}$ & $\begin{array}{c}15 \\
(23)\end{array}$ & $\begin{array}{l}15,8 \\
(28)\end{array}$ & $\begin{array}{c}16,7 \\
(9)\end{array}$ \\
\hline TOTALES & 85 & 161 & 180 & 190 & 190 & 46 & 42 & 152 & 178 & 54 \\
\hline
\end{tabular}

Por último, no hay diferencias estadísticamente significativas por edad. Ello indica que el fenómeno bullying está presente en todas las edades de nuestro estudio. Sin embargo, podemos observar en la tabla 2 como hay un incremento paulatino del porcentaje de implicación, que se hace más notorio en las edades de 10, 11 y 12 años, con un 15\%, 16\% y 17\% respectivamente, y cabe señalar que con la edad crece el porcentaje de sujetos implicados co- 
mo agresores (de 5\% a 11\%) y decrece el porcentaje de sujetos víctimas (de 6\% a 4\%), así como que a la edad de 9 años no encontramos ningún sujeto bajo el perfil de víctimaprovocadora.

Nivel de implicación en bullying y variables personales y sociodemográficas

Tabla 3. Incidencia del bullying. Frecuencias por sexo, n.e.a.e, repetición e inmigración

\begin{tabular}{|c|c|c|c|c|c|}
\hline & \multicolumn{2}{|c|}{ SEXO } & \multirow{2}{*}{ N.E.A.E } & \multirow{2}{*}{ INMIGRACIÓN } & \multirow[b]{2}{*}{ REPETICIÓN } \\
\hline & $\mathrm{H}$ & $\mathrm{M}$ & & & \\
\hline OTROS & $\begin{array}{c}75,4 \\
(156)\end{array}$ & $\begin{array}{c}94,1 \\
(206)\end{array}$ & $\begin{array}{c}66 \\
(12)\end{array}$ & $\begin{array}{l}72,4 \\
(21)\end{array}$ & $\begin{array}{l}72,4 \\
(21)\end{array}$ \\
\hline BULLY & $\begin{array}{l}15,5 \\
(32)\end{array}$ & $\begin{array}{l}0,5 \\
(1)\end{array}$ & $\begin{array}{l}5,6 \\
(1)\end{array}$ & $\begin{array}{c}17,2 \\
(5)\end{array}$ & $\begin{array}{c}10,3 \\
(3)\end{array}$ \\
\hline VICTIMA & $\begin{array}{c}6,8 \\
(14)\end{array}$ & $\begin{array}{l}4,1 \\
(9)\end{array}$ & $\begin{array}{c}22,2 \\
(4)\end{array}$ & - & $\begin{array}{l}6,9 \\
(2)\end{array}$ \\
\hline $\mathrm{V}-\mathrm{P}$ & $\begin{array}{l}2,4 \\
(5)\end{array}$ & $\begin{array}{l}1,4 \\
(3)\end{array}$ & $\begin{array}{l}5,6 \\
(1)\end{array}$ & $\begin{array}{c}10,3 \\
(3)\end{array}$ & $\begin{array}{c}10,3 \\
(3)\end{array}$ \\
\hline TOTAL BULLYING & $\begin{array}{l}24,7 \\
(51)\end{array}$ & $\begin{array}{c}6 \\
(13)\end{array}$ & $\begin{array}{c}33,4 \\
(6)\end{array}$ & $\begin{array}{c}27,5 \\
(8)\end{array}$ & $\begin{array}{c}27,5 \\
(8)\end{array}$ \\
\hline TOTAL & 207 & 219 & 18 & 29 & 29 \\
\hline
\end{tabular}

\section{- Implicación en bullying por sexo}

En relación al sexo, se observa una mayor implicación en el fenómeno bullying por parte de los chicos, ya que éstos se ven implicados en estas dinámicas en un $25 \%$ frente a un $6 \%$ de las chicas. En esta variable, es necesario un análisis por perfiles, ya que las diferencias tan acusadas responden a los sujetos implicados como agresores. Se observa así, como un $15,5 \%$ de ellos son chicos y representan el $97 \%$ de este perfil, frente a un $0,5 \%$ de chicas.

En los perfiles de víctima y víctimas-provocadora, el porcentaje de los chicos sigue siendo superior en ambos casos, aunque las diferencias no son tan acusadas y se observa en la tabla 3 como un $7 \%$ de chicos son víctimas frente al $4 \%$ de chicas y un $2,5 \%$ de chicos son víctimas-provocadoras frente a un 1,5\% de chicas. En este análisis, llama nuestra atención, el hecho de que los chicos representan casi el total de los sujetos implicados como agresores, pero un $40 \%$ de víctimas y un 37,5\% de víctimas-provocadoras son chicas, por lo que las diferencias no están tan marcadas en estos dos perfiles. 
Por último, y en relación a esta variable, cabe resaltar que además de encontrar diferencias estadísticamente significativas $\left(\chi^{2}(3, \mathrm{~N}=426)=37.30, \mathrm{p}<.001\right)$, se observa un rango de significación del 29\% según el coeficiente de Phi y V de Cramer, que nos permite realizar una interpretación práctica entre estas dos variables y afirmar que existe una relación considerable entre el sexo y el fenómeno bullying.

\section{- Implicación en bullying de los sujetos con necesidades específicas de apoyo educativo}

Otra de las variables personales recogidas en el estudio es presentar necesidades educativas específicas de apoyo educativo (n.e.a.e). Como se observa en la tabla 3 , un $33 \%$ de estos sujetos están implicados en la dinámica bullying destacando el hecho de que un $22 \%$ se ven envueltos en este tipo de situaciones como víctimas y sólo un 5,6\% lo hacen como agresores o víctimas-provocadoras, respectivamente.

Es así que, encontramos una relación significativa estadísticamente entre esta variable y el fenómeno bullying $\left(\chi^{2}(3, \mathrm{~N}=426)=12.06, \mathrm{p}<.01\right)$, y una relación a nivel práctico que alcanza un valor estadístico del 17\% (Phi y V de Cramer=.168) que nos permite decir que existe cierta relación entre ser sujeto de necesidades específicas de apoyo educativo y verse implicado en el fenómeno bullying, principalmente como víctima.

\section{- Implicación en bullying de los sujetos inmigrantes}

La siguiente variable personal analizada fue la nacionalidad, estudiada a través de la variable inmigración. En relación a ella, observamos en la tabla 3 como un 27,5\% de los sujetos inmigrantes se ven envueltos en este tipo de situaciones. De los resultados se destaca que los sujetos inmigrantes sólo están implicados como agresores (17\%) y como víctimasprovocadoras (10\%) y que además representan el 37,5\% del total de las víctimasprovocadoras.

En el análisis de estas variables encontramos una relación significativa $\left(\chi^{2}(3, N=426)\right.$ $=17.75, \mathrm{p}<.001$, que además alcanza un rango de significación práctica del 20\%, observándose así que ser inmigrante es un factor de riesgo a tener en cuenta en la dinámica bullying y que incide principalmente sobre los perfiles de agresor y víctima-provocadora. 
En relación, a la variable repetición, observamos en la tabla 3 que un 27,5\% de los sujetos repetidores también se ven implicados en la dinámica bullying y además lo hacen principalmente como agresores y víctimas-provocadoras. Se observa un porcentaje de $10 \%$ para ambos perfiles respectivamente, frente a un $7 \%$ de implicados como víctimas. Al igual que sucede con los sujetos inmigrantes, destaca el porcentaje elevado en el perfil de víctimasprovocadoras, representando un $37,5 \%$ de este perfil.

La relación entre estas dos variables es estadísticamente significativa $\left(\chi^{2}(3, \mathrm{~N}=426)\right.$ $=12.85, \mathrm{p}<.01)$ y además encontramos una significación práctica que alcanza el 17\% (Phi y $\mathrm{V}$ de Cramer $=0.174$ ) por lo que el efecto repetidor incide de manera considerable en el fenómeno bullying, convirtiéndose en un factor de riesgo para verse implicado en bullying, en nuestro caso, principalmente, como agresor y víctima-provocadora.

\section{Características personales y su relación con el rechazo social}

En el análisis de las variables personales también hemos querido ver si existe relación entre éstas y los aspectos sociales. Los datos obtenidos ponen de manifiesto que existe una baja integración social de estos sujetos en el aula, que sin duda, está relacionada con su implicación en bullying, tal como se observa en la tabla 4 .

Los sujetos de necesidades educativas específicas de apoyo educativo son rechazados por el grupo $(\mathrm{M}=12.16, \mathrm{DT}=15.14)$, a la vez que también son menos elegidos por los compañeros $(\mathrm{M}=1.61$, DT $=2.56)$ presentando las diferencias de medias más altas en estas dos variables con respecto a los otros como se puede observar en la tabla $4(\mathrm{p}=.03 ; \mathrm{p}<.01)$. Además, se puede apreciar como ellos mismos presentan una baja expectativa de ser elegidos $(p<.001)$ y se encuentran aislados dentro del aula.

Los sujetos inmigrantes también presentan medias altas en rechazo $(\mathrm{M}=9.72, \mathrm{DT}=$ 14.06) y bajas en número de elecciones $(\mathrm{M}=1.75$; $\mathrm{DT}=2.16)$ observándose diferencias de medias significativas con respecto a los otros en ambas variables $(p=.02 ; p<.001)$. Además, este grupo de sujetos presenta una baja expectativa de ser elegido $(\mathrm{p}<.001)$ y una alta expecta- 
tiva de ser rechazado $(\mathrm{p}=.02)$, siendo más conscientes de su situación de aislamiento y rechazo dentro del aula.

Tabla 4: Diferencias T-Test Bull-S entre otros/ n.e.a.e / inmigrante/ repetetido en rechazo y aceptación

\begin{tabular}{|c|c|c|c|c|c|c|c|c|c|}
\hline & \multicolumn{3}{|c|}{ OTROS/N.E.A.E } & \multicolumn{3}{|c|}{ OTROS/INMIGRANTE } & \multicolumn{3}{|c|}{ OTROS/REPETIDOR } \\
\hline & $\mathbf{T}$ & G.L & DIF.M & $T$ & G.L & DIF.M & $\mathbf{T}$ & G.L & DIF.M \\
\hline RECHAZO & $-2,37^{* *}$ & 17,37 & -850 & $-2,32^{* *}$ & 29,13 & $-6,12$ & $-2,80^{* * *}$ & 29,48 & $-6,64$ \\
\hline ELEGIDO & $3,13^{* * *}$ & 424 & 3,30 & $7,02^{* * *}$ & 47,72 & 3,23 & $4,49^{* * *}$ & 38,19 & 2,64 \\
\hline $\begin{array}{l}\text { EXPECTATIVA } \\
\text { ELEGIDO }\end{array}$ & $5,89^{* * *}$ & 22,04 & 2,64 & $6,43^{* * *}$ & 39,15 & 2,59 & $3,57^{* * *}$ & 424 & 2,14 \\
\hline $\begin{array}{l}\text { EXPECTATIVA } \\
\text { RECHAZO }\end{array}$ & - & - & - & $-2,44^{* *}$ & 30,68 & -279 & $-276^{* * *}$ & 424 & $-2,60$ \\
\hline
\end{tabular}

P. Asociada: ${ }^{*} \mathrm{p}<.05 ; * * \mathrm{p}<.01 ; * * * \mathrm{p}<.001$

Las medias de los sujetos repetidores también muestran puntuaciones altas en rechazo $(\mathrm{M}=10.20$; D.T. $=12.59)$ y bajas en número de elecciones $(\mathrm{M}=2,31, \mathrm{DT}=2.92)$ con respecto a los otros, encontrando diferencias significativas en ambas variables $(p<.01 ; p<.001)$. Además sus expectativa de ser elegido $(\mathrm{p}<.001)$ y de ser rechazado $(\mathrm{p}<.01)$ también muestran que este grupo de sujetos presentan aspectos psicosociales bastante negativos.

\section{Discusión y Conclusiones}

El estudio comprueba que las situaciones bullying se están produciendo de forma indistinta en todos los grupos de los últimos niveles de Educación Primaria, siendo su incidencia significativa en edades más tempranas en las que el entramado relacional y social tiende a consolidarse (Eisenbraun, 2007), por lo que la edad no parece ser un factor relevante en la incidencia del fenómeno.

En el análisis de la incidencia por sexo los resultados obtenidos coinciden con los numerosos estudios que confirman que el sexo es un factor de riesgo para la implicación en bullying, siendo éste un fenómeno preferentemente masculino (Cerezo, 2009; Seals y Young, 2003; Solberg y Olweus, 2003; Tapper y Boulton, 2004). Los datos muestran que el perfil de agresor se ve prácticamente representado por chicos, apuntando ciertos estudios a la relación 
entre ser chico y tener baja competencia social a la vez que restan importancia a este tipo de situaciones y están más habituados a las conductas agresivas (Avilés, 2006; Trianes, CardelleElawar, Blanca y Muñoz, 2003).

Sin embargo, encontramos que las chicas alcanzan porcentajes considerables en el perfil de víctimas y víctimas-provocadoras, comprobándose que la tendencia a que éste sea un fenómeno casi exclusivo del género masculino está cambiando (Carney y Merrell, 2001; Benítez y Justicia; 2006). Estos resultados pueden estar indicando que las conductas agresivas de las chicas, lejos de ser infrecuentes, pueden estar pasando más desapercibidas por tratarse de agresiones indirectas cuya observación y detección se hace más difícil a la vez que resultan más difíciles de identificar por el alumnado (González, Postigo, Montoya y Martorell, 2007; Toldos, 2005; Veenstra et al., 2005).

Los datos nos indican que ser un sujeto con necesidades específicas de apoyo educativo se relaciona significativamente con la implicación en bullying, siendo esta característica un factor de riesgo que incide principalmente en el perfil de víctima. En esta línea, consideramos interesante seguir profundizando y poder asociar ciertas necesidades educativas a cada perfil, ya que como señalan algunos estudios (Farrigton, 2005; Unnever y Cornell, 2003) los sujetos con necesidades por déficit de atención e hiperactividad actúan de forma agresiva para defenderse por lo que se verían más implicados en el perfil de víctimas-provocadoras.

En relación a la nacionalidad los resultados coinciden con los obtenidos por Unnever y Cornel (2003) e indican que ser un sujeto inmigrante es un factor de riesgo que está relacionado con la incidencia del fenómeno bullying. Además, dado el número de sujetos inmigrantes en nuestros centros educativos creemos interesante seguir investigando en esta línea y profundizar en otros aspectos que pueden estar relacionados con su implicación en bullying, tales como la nacionalidad o el momento de incorporación a nuestro sistema educativo.

En relación al efecto repetición, los resultados obtenidos reflejan una relación significativa entre ser repetidor y verse implicado en la dinámica bullying bajo el perfil de agresor (Cerezo, 2006), aunque el estudio también revela un alto número de sujetos repetidores que se ven implicados como víctimas-provocadoras, por lo que esta medida puede tener una doble cara. Desde este punto de vista, es importante considerar los aspectos sociales en las decisiones de repetición de los alumnos en la etapa de Educación Primaria. 
Por último, como prospectiva del estudio se plantea la comprensión del fenómeno bullying desde el planteamiento de una teoría cognitivo-relacional que considere las características individuales de los sujetos y la red de iguales como unidades de relación (Ortega y Mora, 2008). Se debe abordar el fenómeno bullying desde un enfoque multicausal, que no sólo se circunscriba a las conductas de los sujetos y a la falta de habilidades sociales o competencia social, sino que preste atención a los aspectos curriculares y organizativos que, sin duda, favorecen una adecuada atención educativa a la diversidad del alumnado y favorecen la integración social de todos y cada uno de ellos (Ballester y Arnaíz, 2001). Desde esta perspectiva es fundamental la labor del centro escolar, como agente educador de todos sus alumnos, ofreciendo un entorno de aprendizaje y convivencia (Ballester y Calvo, 2007).

\section{Referencias}

Avilés, J. y Monjas, I. (2005). Estudio de la incidencia de la intimidación y el maltrato entre iguales en la Educación Secundaria Obligatoria mediante el cuestionario CIMEI (Cuestionario sobre Intimidación y Maltrato Entre Iguales). Anales de Psicología, 21(1), 27-41.

Avilés, J. M. (2006). Diferencias de atribución causal en el bullying entre sus protagonistas. Electronic Journal of Research in Educational Psychology, 9, 4(2), 201-220.

Ballester, F. y Arnaiz. P (2001). Diversidad y violencia escolar. Revista Interuniversitaria de Formación del profesorado, 41, 39-58

Benítez, J.L y Justicia, F. (2006). El maltrato entre iguales: descripción del fenómeno. Electronic Journal of Research in Educational Psychology, 9, 4(2), 151-170.

Card, N. A. (2003). Victims of peer aggression: A meta-analytic review. Presented at Society for Research in Child Development biennial meeting, Tampa, USA, April

Carney, A. G. y Merrell, K. W. (2001). Bullying in schools: Perspectives on understanding and preventing an international problem. School Psychology International, 22(3), 364382.

Cerezo, F. (2002). BULL-S: Test de evaluación de la agresividad entre escolares. Manual de referencia. Bizcaia: Grupo ALBOR-COHS.

Cerezo, F. (2006). Violencia y victimización entre escolares. El bullying: Estrategias de identificación y elementos para la intervención a través del Test Bull-S. Electronic Journal of Research in Educational Psychology, 9, 4(2), 333-352. 
Cerezo, F (2009). Análising bullying in Spanish Schools. International Journal of psychology and psychological therapy, 9(3), 383-394.

Cerezo, F. (2010). Social status, gender, classroom climate and bullying among adolescents pupils. Anales de psicología, 26(1), 137-144.

Cerezo, F., Calvo, A., y Sánchez, C. (2004). Bullying y estatus social en el grupo-aula en una muestra de escolares. Comunicación presentada al IV Congreso Internacional de Psicología y Educación. Almería, 30 de Marzo al 2 de Abril de 2004. Actas del Congreso.

Craig, W. y Pepler, D. (2007). Understanding bullying: From research to practice. Canadian Psychology, 48(2), 86-93.

Defensor del Pueblo (2007). Violencia Escolar: El maltrato ente Iguales en la Educación Obligatoria Secundaria 1999-2006. Madrid: Publicaciones de la Oficina del Defensor del Pueblo.

Díaz-Aguado, M. J. (2005). La violencia entre iguales en la adolescencia y su prevención desde la escuela. Psicothema, 17(4), 549-558.

Eslea, M. y Rees, J. (2001). At what age are children most likely to be bullied at school? $A g$ gressive Behavior, 27(6), 419-429.

Eisenbraun, K. D. (2007). Violence in schools: Prevalence, prediction, and prevention. Aggression and Violent Behavior, 12(4), 459-469.

Farrington, D. P. (2005). Childhood origins of antisocial behavior. Clinical Psychology y Psychotherapy, 12(3), 177-190.

Gifford-Smith, M. y Brownell, C. (2003). Childhood peer relationships: Social acceptance, friendships, and peer networks. Journal of School Psychology, 41(4), 235-284.

González, R., Postigo, S., Montoya, I. y Martorell, M. C. (2007). Implicación femenina en el acoso escolar. En Gázquez, J. J., Pérez, Ma . C., Cangas, A. J. y Yuste, N. (eds.), Situación actual y características de la violencia escolar, Vol. I, 179-184. Almería: Grupo Editorial Universitario

Mestre, M. V., Samper, P. y Frías, M. D. (2002). Procesos cognitivos y emocionales predictores de la conducta prosocial y agresiva: La empatía como factor modulador. Psicothema, 14(2), 227-232.

Montero, I. y León, O. G. (2007). Guía para nombrar los estudios de investigación en Psicología. International Journal of Clinical and Health Psychology, 7(3), 843-862.

Ortega, R. y Mora, J. (2008). Las redes de iguales y el fenómeno del acoso escolar: explorando el esquema dominio-sumisión. Infancia y aprendizaje, 31(4), 515-528. 
Postigo, S., González, R., Mateu, C., Ferrero, J. y Martorel, C. (2009). Diferencias conductuales según género en convivencia escolar. Psicothema, 21(3), 453-458.

Seals, D. y Young, J. (2003). Bullying and victimization: Prevalence and relationship to gender, grade level, ethnicity, self-esteem, and depression. Adolescence, 38(152), 735 747.

Serrano, A. e Iborra, I. (2005). Violencia entre compañeros en la escuela. Centro Reina Sofía para el Estudio de la Violencia. Valencia: Goeprint

Smith, P. K. (2004). Bullying: Recent developments. Child y Adolescent Mental Health, 9(3), 98-103.

Solberg, M. E. y Olweus, D. (2003). Prevalence estimation of school bullying with the Olweus Bully/Victim questionnaire. Aggressive Behavior, 29(3), 239-268.

Tapper, K. y Boulton, M. J. (2004). Sex differences in levels of physical, verbal, and indirect aggression amongst primary school children and their associations with beliefs about aggression. Aggressive Behavior, 30(2), 123-145.

Toldos, M. P. (2005). Sex and age differences in self-estimated physical, verbal and indirect aggression in spanish adolescents. Aggressive Behavior, 31(1), 13-23.

Trianes, Ma . V., Blanca, Mª J., García, J. y Sánchez, A. (2003). Competencia social en alumnos con N.E.E: Nivel de inteligencia, edad y género. Revista de Psicología General y Aplicada, 56(3), 325-338.

Trianes, Ma. V., Cardelle-Elawar, M., Blanca, Ma. J. y Muñoz, A. (2003). Contexto social, género y competencia social autoevaluada en alumnos andaluces de 11-12 años. Electronic Journal of Research in Educational Psychology, 1(2), 37-55.

Unnever, J. D. y Cornell, D. G. (2003). Bullying, self-control, and ADHD. Journal Interpersonal Violence, 18(2), 129-147.

Veenstra, R., Lindenberg, S., Oldehinkel, A. J., De Winter, A. F., Verhulst, F. C. y Ormel, J. (2005). Bullying and victimization in elementary schools: A comparison of bullies, victims, Bully/Victims, and uninvolved preadolescents. Developmental Psychology, 41(4), 672-682.

Wolke, D., Woods, S., Stanford, K. y Schulz, H. (2001). Bullying and victimization of primary school children in England and Germany: Prevalence and school factors. British Journal of Psychology, 92(4), 673.

Woods, S., Hall, L., Dautenhahn, K. y Wolke, D. (2007). Implications of gender differences for the development of animated characters for the study of bullying behavior. Computers in Human Behavior, 23(1), 770-786. 